\title{
3D Particle Shape of Calcareous Sand Conducted by X-Ray Computed Tomography
}

\author{
Pham Huu Ha Giang¹, Peter Van Impe ${ }^{2}$, William Van Impe ${ }^{1,2}$, Patrick Menge ${ }^{3}$, Veerle Cnudde ${ }^{4}$, Wim \\ Haegeman $^{1}$ \\ ${ }^{1}$ Department of Civil Engineering, Ghent University \\ Technologiepark 905, 9052 Zwijnaarde, Belgium \\ huuhagiang.pham@ugent.be; wim.haegeman@ugent.be \\ ${ }^{2}$ AGE Advanced Geotechnics Engineering Bvba \\ Rooseveltlaan 2, 9420 Erpe-Mere, Belgium \\ peter.vanimpe@age-be.net; william.vanimpe@age-be.net \\ ${ }^{3}$ Dredging International \\ Scheldedijk 30, 2070 Zwijndrecht, Belgium \\ menge.patrick@deme.be \\ ${ }^{4}$ Department of Geology and Soil Science, Ghent University \\ Krijgslaan 281, S8 9000, Gent, Belgium \\ veerle.cnudde@ugent.be
}

\begin{abstract}
The paper presents the analysis of 3D particle shape on three-grain fractions of a calcareous sand based on the raw data of $\mathrm{X}$-Ray computed tomography (X-Ray CT). The results show that the larger fraction size is found to have less spheres and rods, and more discs than the smaller one, and there is a significant difference in grain size obtained by 3D analysis and sieve analysis. The difference becomes greater in the larger fraction size, and 3D analysis gives smaller values of particle size than sieve analysis. This proves that particle shape is a very important factor influencing the grain size distribution of calcareous sand.
\end{abstract}

Keywords: 3D analysis, calcareous sand; grain size distribution, shape index, sphericity

\section{Introduction}

In an attempt to define particle shape, Wadell [1], a pioneer investigated the particle shape of rock, defined that sphericity (SPH) is the ratio of the surface area of a sphere with the same volume as the particle to the actual surface area of the particle, and roundness is the ratio of the average radius of curvature of the corners to the maximum inscribed circle. From his study, the sphericity is found to be entirely independent of roundness.

In general, particle shape has three features including form, roundness and surface texture shown in Fig. 1 [2]. While the roundness reflects the sharpness in corners and edges of grain, the particle form is simply understood as its geometric form determined by the relative length of the particle intercepts along the three perpendicular axes corresponding to three orthogonal axes $\mathrm{X}$, Y, and Z.

Up to now, numerous sphericity-form diagrams of particle shape classification have been suggested for describing particle form based on the relation of three orthogonal axes of particles [3-5]. For this approach, the particle form is divided into four types of particle shape (spheres, discs, rods and blades) and is related to shape index. The usage of the sphericity-form diagrams completely depends on an author's definitions of shape index and sphericity. Among these diagrams, the sphericity-form diagram of Illenberger [5] became more popular because it is consistent with field particle shapes.

The dependence of sphericity on particle size was discussed by Pettijohn and Lundahl [6]. Their results showed a significant difference in grain size; the larger size was the most spherical. However, Cho et al. [7] and Das and Ashmawy [8] confirmed no relationship between grain size and grain shape. Recently, Dewen et al. [9] stated that the sphericity increased with a decreasing particle size. In addition, the relationship between roundness and particle size was found not 
evident in previous studies. The larger particle size was much rounder than the smaller one [6,10,11]. In contrast, such an observation from some researchers confirmed that the finer particle was the more rounded particle $[12,13]$.

In order to measure particle shape, 2D analysis is commonly used to determine directly the roundness of particle, which is estimated based on the visual 2D plane image of grain obtained by microscope. Currently, with the development of technology and the digital image processing (DIP) technique in the 2D image, scientists are able to effectively estimating thickness and volume of particles. Although 2D image analysis is easy to perform; due to the difference in materials and the variation in particle shape, the application of silica shape charts based on 2D plane images to determine the degree of shape for calcareous sand particles is unrealistic and unreasonable. In recent years, several techniques have been developed to provide opportunities for identifying particle morphology in 3D such as automated serial sectioning tomography, scanning transmission electron microscopy tomography, and X-ray micro computed tomography.

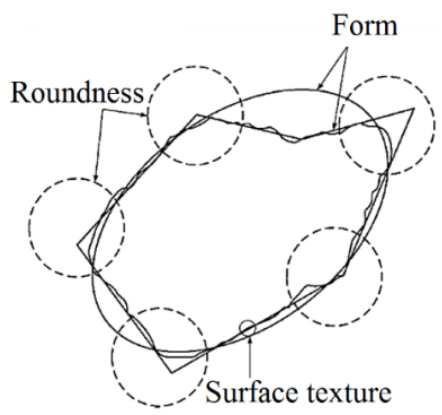

Fig. 1: Particle shape (form, roundness, and surface texture) proposed by [2].

In this study, 3D analysis of particle shape using X-Ray micro-computed tomography (X-Ray CT) is performed to evaluate the relationship between particle size and particle shape for calcareous sand. The particle size and shape indices such as Corey shape index (CSI), disc-rod index (DRI) and sphericity (SPH) of calcareous sand are presented and discussed.

\section{Materials}

The calcareous sand, Sarb sand, used in this study was obtained from an artificial island in the United Arab Emirates. 3D particle analysis is performed on three size fractions obtained from three sieves: No. $90: 160 \mu \mathrm{m}-250 \mu \mathrm{m}$

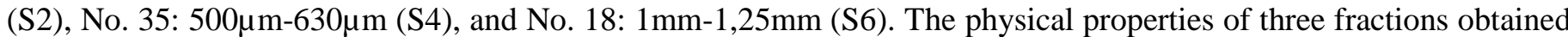
from Sarb sand are summarized in Table 1.

Table 1: Physical properties of three fractions of Sarb sand obtained by sieve analysis.

\begin{tabular}{|l|c|c|c|}
\hline Physical properties & $\mathrm{S} 2$ & $\mathrm{~S} 4$ & S6 \\
\hline Sieve size $(\mathrm{mm})$ & $0.16-0.25$ & $0.5-0.63$ & $1-1.25$ \\
\hline Mean grain size $(\mathrm{mm})$ & 0.205 & 0.565 & 1.125 \\
\hline Maximum void ratio, $\mathrm{e}_{\max }$ & 1.376 & 1.556 & 1.835 \\
\hline Minimum void ratio, $\mathrm{e}_{\min }$ & 0.933 & 1.042 & 1.224 \\
\hline
\end{tabular}

\section{Methodology}

The procedure of 3D measurement and analysis consists of scanner operation and control, tomographic reconstruction, and 3D analysis and visualization (Octopus). The scanner operation and control are performed at the center for X-ray tomography of Ghent University (UGCT). The whole system includes hardware for scanning and data acquisition, software tools for reconstructing radiographs and 3D visualization.

$\mathrm{X}$-ray computed tomography is a non-destructive technique for visualizing the internal structure of objects and for obtaining 3D information on their shape. In this study, the X-ray computed tomography device used is a highresolution X-ray CT (HRXCT) scanner developed at UGCT. The source-detector is fixed while the object, which is 
placed on a rotary stage (rotation angles from $0^{\circ}$ to $360^{\circ}$ ) between the source and the detector, is rotated during scanning (Fig. 2).

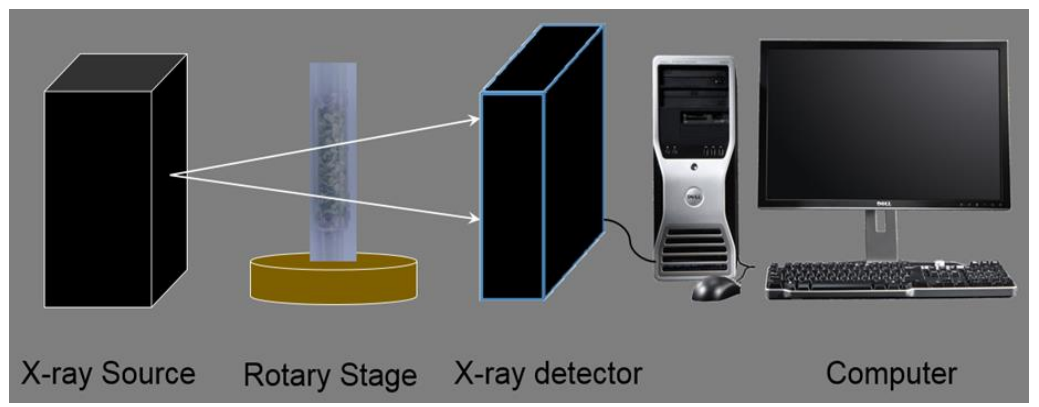

Fig. 2: Concept of X-ray CT scan.

Octopus formerly known as Morpho+ is a tool to determine and select a volume of interest (VOI) and has been developed at Ghent University. Octopus allows for the determination of the porosity and the volume fraction of a component in the dataset [14]. Over a few years, the program has been modified and extended to keep up with the demands of its users and is now continuously upgraded. There are two separate packages, Octopus analysis and viewer. In 3D Octopus viewer, the database of 3D analysis is visualized, in which the particles are rendered in arbitrary colors to present the contrast among individual particles with the different colors showing a variation of sphericity (SPH). For 3D Octopus analysis, some definitions are used as follows:

- Maximum opening is the diameter of the largest inscribed sphere in an object, and minimum closing (MC) is the diameter of the smallest circumscribed sphere in an object (Fig. 3).

- The equivalent diameter is defined as the diameter of a sphere with the number of voxels inside an object.

- The equivalent ellipsoid, which has the same moments of inertia as the object, is used to calculate the disc-rod index and shape index.

- The sphericity (SPH) is defined as the ratio of the maximum opening and the equivalent diameter.

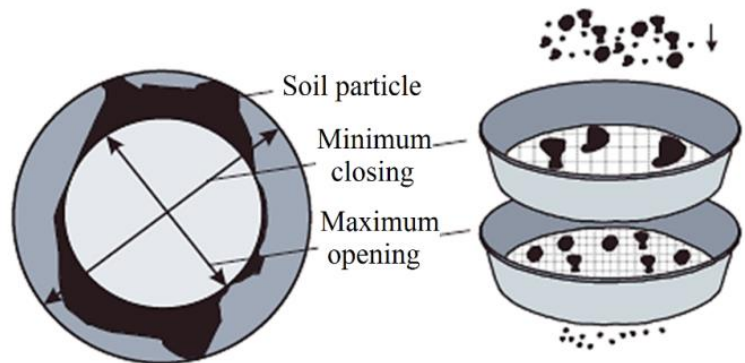

Fig. 3: Maximum opening and minimum closing of a particle and the corresponding sieves [14].

The flowchart of 3D particle analysis consists of load volume, filter, threshold, fill holes, labeling, distance map, separate object, and re-join. A typical result is shown as in Fig. 4.

The analysis results are plotted on the diagram proposed by Illenberger [5]. In these diagrams, the Corey shape index $\left(\mathrm{CSI}=\mathrm{S} /(\mathrm{IL})^{1 / 2}\right)$ is plotted versus the disc-rod index $(\mathrm{DRI}=(\mathrm{L}-\mathrm{I}) /(\mathrm{L}-\mathrm{S}))$; where $\mathrm{L}, \mathrm{I}$, and $\mathrm{S}$ refer to the lengths of the longest, intermediate, and shortest axes, respectively. The output data files can be extracted by using MATLAB and transferred to Excel to perform analysis efficiently. 

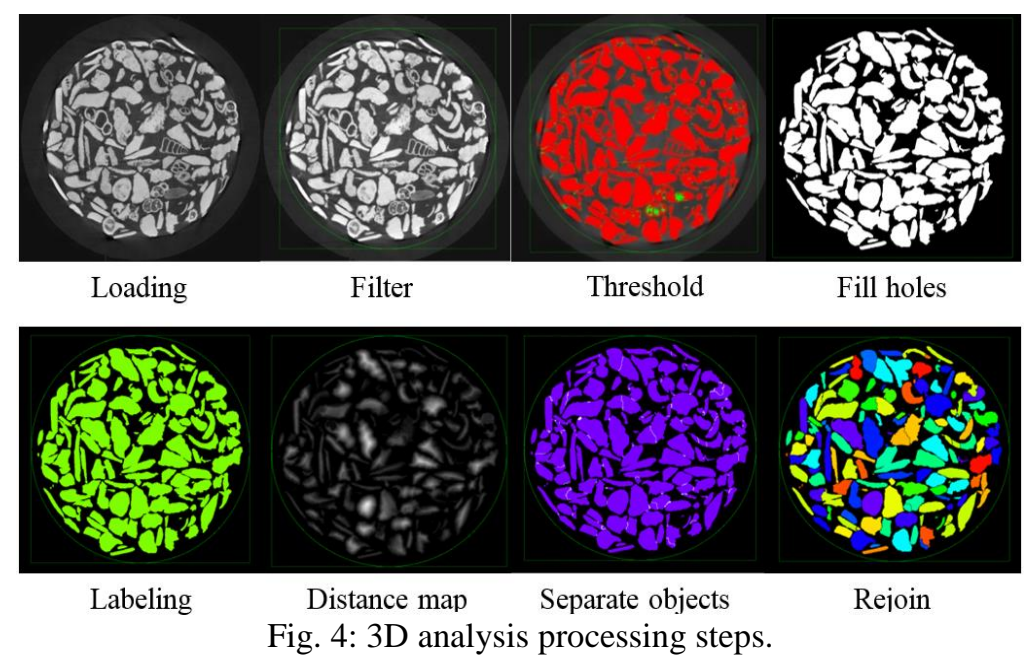

\section{Results and discussion}
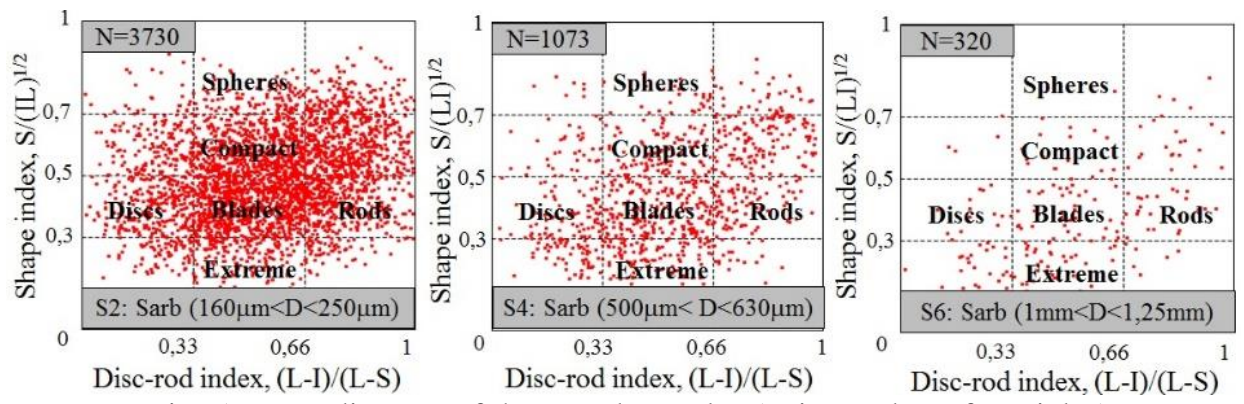

Fig. 5: Form diagram of the tested samples ( $\mathrm{N}$ is number of particles).

The particle shape distributions of three samples are plotted on the form diagrams with the shape index versus the disc-rod index shown in Fig. 5. Because the particle number in each sample is different causing a particular difficulty in discriminating the difference among these samples. Therefore, the percentages of particle shapes in each sample are demonstrated as in Error! Reference source not found.. In this figure, the blades appear the most followed by rods, discs, spheres. the larger fraction is found to have less spheres and rods, and more discs than the smaller one. In addition, the average sphericity (SPH) obtained by 3D analysis (3DA) and the mean grain size obtained by sieve analysis (SA) are illustrated in Fig. 7. It can be seen from the figure that SPH decreases with an increasing grain size.

In this study, the 3DA is compared with the sieve analysis (SA). It is clear that there is a significant difference in grain size obtained by the two methods (Fig. 8). The 3DA gives smaller values of particle size than the SA. The difference between the results of SA and 3DA is due to the effect of particle shape, namely some particles tries to pass through a sieve by longest dimension and particles retained on a sieve due to various particle shapes [15]. In this case, it can be concluded that the particle shape has an effect on the particle size obtained by the SA. This effect becomes smaller in the samples of the smaller fraction size. Furthermore, the range of void ratio in a grain soil mass depends on the shape of particles as well as the grain size distribution. Indeed, the determination of maximum and minimum void ratios is considered as an alternative method to see the effect of particle shape on density in this study. Table 1 shows that the void ratios of the larger fraction size are higher than that of the smaller one. This is a reasonable result and once again re-affirm the results of 3DA is consistent with reality as the void ratios ( $e_{\max }$ and $\left.e_{\min }\right)$ decrease with increasing sphericity.

$3 \mathrm{D}$ view is considered the easiest and the most realistic way to determine particle shape related to the average values of SPH in each sample. Fig. 9 demonstrates the 3D rendered images of three tested samples with different SPH values in different colors throughout each sample. 


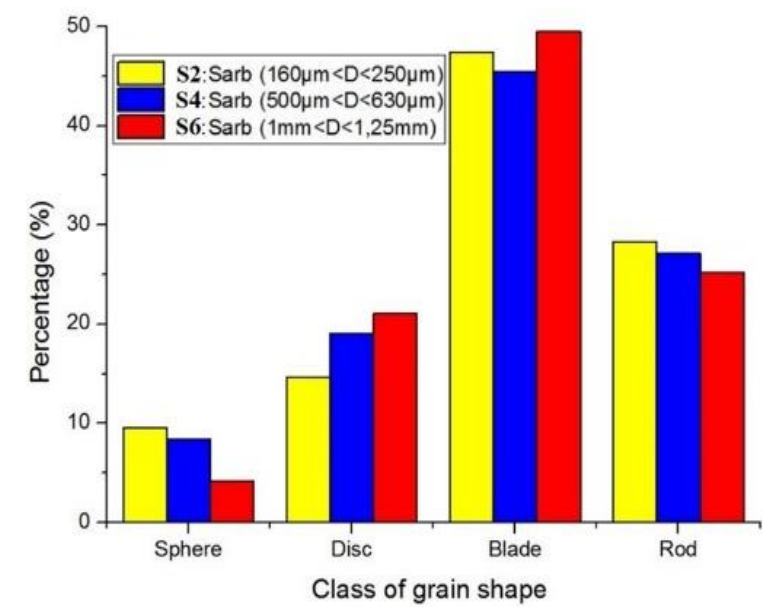

Fig. 6: Percentage of grain shape in different particle size.

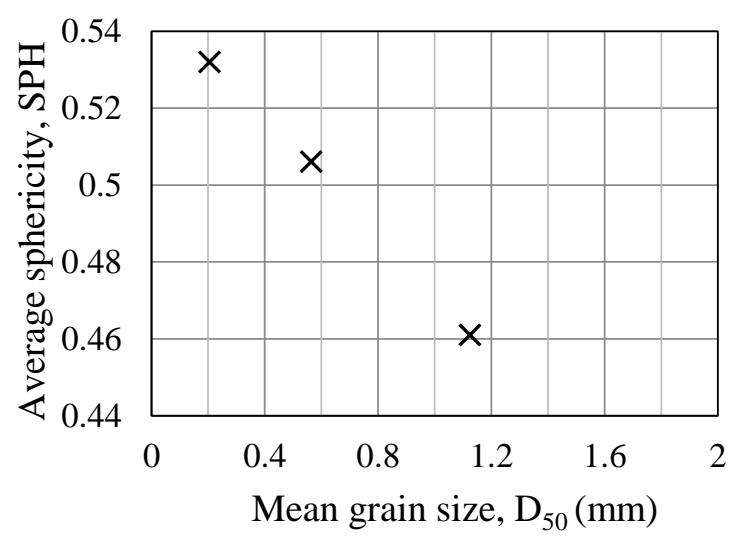

Fig. 7: Relationship between sphericity and mean grain size.

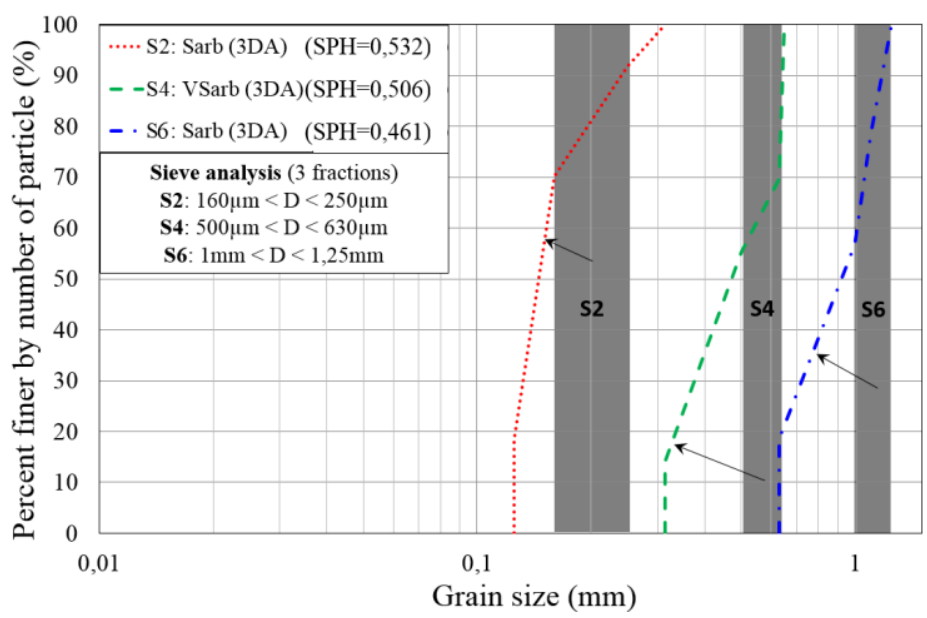

Fig. 8: Percentage of grain size in the samples. 


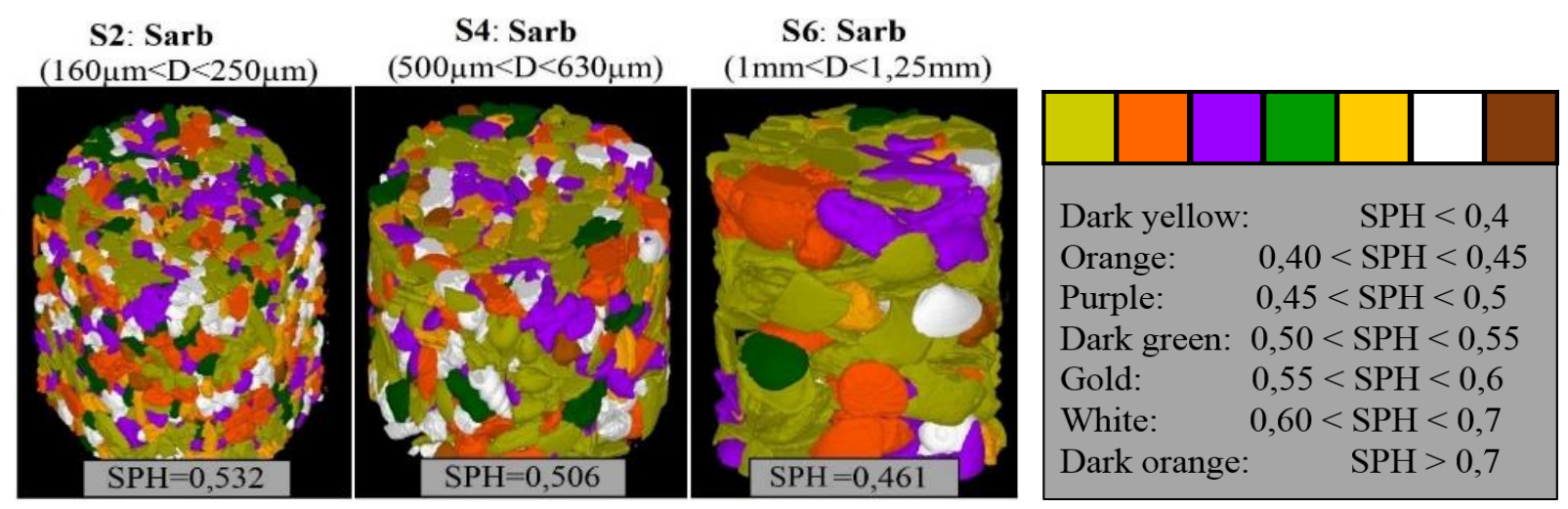

Fig. 9: Simulated samples rendered by 3D Octopus viewer.

\section{Conclusion}

The 3D particle shape of calcareous sand is simulated in this study based on the raw data of X-Ray micro-computed tomography (X-Ray CT) and 3D analysis software. The 3D analysis can provide much more information about the particle shape and size than the sieve analysis. The morphology of the particles is affected by the classification of particle size in the sieve analysis (SA). It can be concluded that various particle characteristics like calcareous sand are more influential on the difference between 3DA and SA. The relationship is found between the particle shape and the particle size. The large particle size has less sphericity than the smaller particle size.

\section{Acknowledgements}

The authors would like to express their gratitude to Dr. T. De Kock, Department of Geology and Soil Science, Ghent University for his kind help in conducting part of the X-Ray Computed Tomography scans. We also would like to acknowledge Erasmus Mundus Action 2, Lotus Project for the financial support.

\section{References}

[1] H. Wadell, "Volume, shape, and roundness of quartz particles," J. Geol., vol. 43, no. 3, pp. 250-280, 1932.

[2] P. J. Barrett, "The shape of rock particles: a critical review," J. Sedimentology, vol. 27, no. 3, pp. 291-303, 1980.

[3] T. Zingg, "Beitrag zur Schotteranalyse," Ph.D. dissertation, Diss. Naturwiss. ETH Zürich, Nr. 849, 1935.

[4] W. C. Krumbein, "Measurement and geological significance of shape and roundness of sedimentary particles," $J$. Sediment Res., vol. 11, no. 2, pp. 64-72, 1941.

[5] W. K. Illenberger, "Pebble shape (and size!)," J. Sediment Res., vol. 61, no. 5, pp. 756-767, 1991.

[6] F. J. Pettijohn and A. C. Lundahl, "Shape and roundness of Lake Erie beach sands," J. Sediment Res., vol. 13, no. 2, pp. 69-78, 1943.

[7] N. Das and A. K. Ashmawy, "Relationship between grain size and shape of natural and crushed sand," Adv. Meas. Model Soil Behav., pp. 1-10, 2007.

[8] G. C. Cho, J. Dodds, and J. C. Santamarina, "Particle shape effects on packing density, stiffness, and strength: natural and crushed sands," J. Geotech. Geoenvironmental Eng., vol. 132, no. 5, pp. 591-602, 2006.

[9] L. Dewen, K. Yanrui, L. Dawei, "Comparison of grain-size and grain-shape characters of alluvial and lakeshore sands based on dynamic image analysis," J. Quat. Sci., vol. 35, no. 2, pp. 484-492, 2015.

[10] W. C. Krumbein, "The effects of abrasion on the size, shape, and roundness of rock fragments," J. Geology, vol. 49, no. 5, pp. 482-520, 1941.

[11] M. R. H. Ramez and F. H. Mosalamy, "The deformed nature of various size fractions in some clastic sands," $J$ Sediment Res., vol. 39, no. 3, pp. 1182-1187, 1969.

[12] I. Banerjee, "Size-roundness relation in the Barakar sandstones of the South Karanpura Coalfield, India," $J$. Sedimentology, vol. 3, no. 1, pp. 22-28, 1964.

[13] J. J. Kasper-Zubillaga, "Roundness in quartz grains from inland and coastal dune sands," Bol. La. Soc. Geol. Mex., vol. 61, pp. 1-12, 2009. 
[14] V. Cnudde, J. Dewanckele, W. De Boever, L. Brabant, and T. De Kock, "3D characterization of grain size distributions in sandstone by means of X-ray computed tomography," in Quant. Mineral Microanal. Sediments Sedimentary Rocks., 2012, pp. 99-113.

[15] S. Arasan, S. Akbulut, and A. S. Hasiloglu, "Effect of particle size and shape on the grain-size distribution using image analysis," Int. J. Civ. Struct. Eng., vol. 1, no. 4, pp. 968-985, 2011.

[16] G. H. A. Kumara, K. Hayano, K. Ogiwara, "Image analysis techniques on the evaluation of particle size distribution of gravel,” Int. J. GEOMATE, vol. 3, no. 1, pp. 290-297, 2012.

[17] C. Mora, A. Kwan, and H. Chan, "Particle size distribution analysis of coarse aggregate using digital image processing," J. Cem. Concr. Res., vol. 28, no. 6, pp. 921-32, 1998. 